\title{
Medicare's per-Beneficiary Potentially Avoidable Admission Measures Mask True Performance
}

\author{
William B. Weeks, MD, PhD, MBA ${ }^{7}$ and James N. Weinstein, DO, MS ${ }^{1,2}$
}

${ }^{1}$ Microsoft Healthcare NExT, Redmond, WA, USA; ${ }^{2}$ The Dartmouth Institute for Health Policy and Clinical Practice, Lebanon, NH, USA.

$J$ Gen Intern Med 35(4):1348-51

DOI: $10.1007 / \mathrm{s} 11606-019-05354-3$

(c) Society of General Internal Medicine 2019

\section{INTRODUCTION}

The Agency for Healthcare Research and Quality's prevention quality indicators (PQIs) use hospital discharge data to identify admissions for ambulatory care-sensitive conditions (ACSCs). ${ }^{1}$ Because they might have been avoided with better access to primary care resources, ACSC admissions are widely considered potentially avoidable, and, therefore, wasteful., ${ }^{2,3}$

Designed to reflect area-level performance, PQIs are calculated using the covered population as the denominator. Because the proportion of admissions that are for ACSCs might better articulate the magnitude of potential waste, ${ }^{3}$ we sought to calculate those proportions and compare them to per-capita rates.

\section{METHODS}

From the Centers for Medicare and Medicaid Services (CMS), for each year (2008-2017) and 2 age groups (< 65 and 65+), we obtained the following: the distribution of Medicare fee-for-service enrollees across age groups; the number of fee-for-service Medicare beneficiaries

Received June 28, 2019

Accepted September 11, 2019

Published online October 21, 2019 who were fully enrolled in Medicare Parts A \& B (without gaps, throughout the year); the number that had a covered inpatient stay; the mean number of stays that those inpatient-using beneficiaries had; hospital readmission rates; and numbers of 8 PQI-defined ACSC admissions per 100,000 age-group-specific beneficiaries (long-term diabetes complications, asthma or COPD, hypertension, congestive heart failure, dehydration, bacterial pneumonia, urinary tract infection, and lower extremity amputation). ${ }^{4}$

For each year and age group, we calculated the total number of inpatient and ACSC-specific admissions as well as the ACSC-specific and overall ACSC per-capita admission rates (aggregating ACSC admissions for 65-74 and 75+ age groups). We also calculated compound annual growth rates (CAGRs) for those measures. This work used publicly available data and was determined to be "Not Human Subjects Research" by Dartmouth College's Committee for the Protection of Human Subjects (CPHS00028121).

\section{RESULTS}

Over the 10-year period, the number of Medicare fee-forservice beneficiaries aged $<65$ increased until 2012 and then decreased; those aged $65+$ generally slowly increased (Table 1). In contrast, the number and proportion of fee-forservice beneficiaries with an inpatient admission fell for both groups, and the number of per-beneficiary inpatient admissions fell in the $65+$ group. Overall, the number of inpatient 


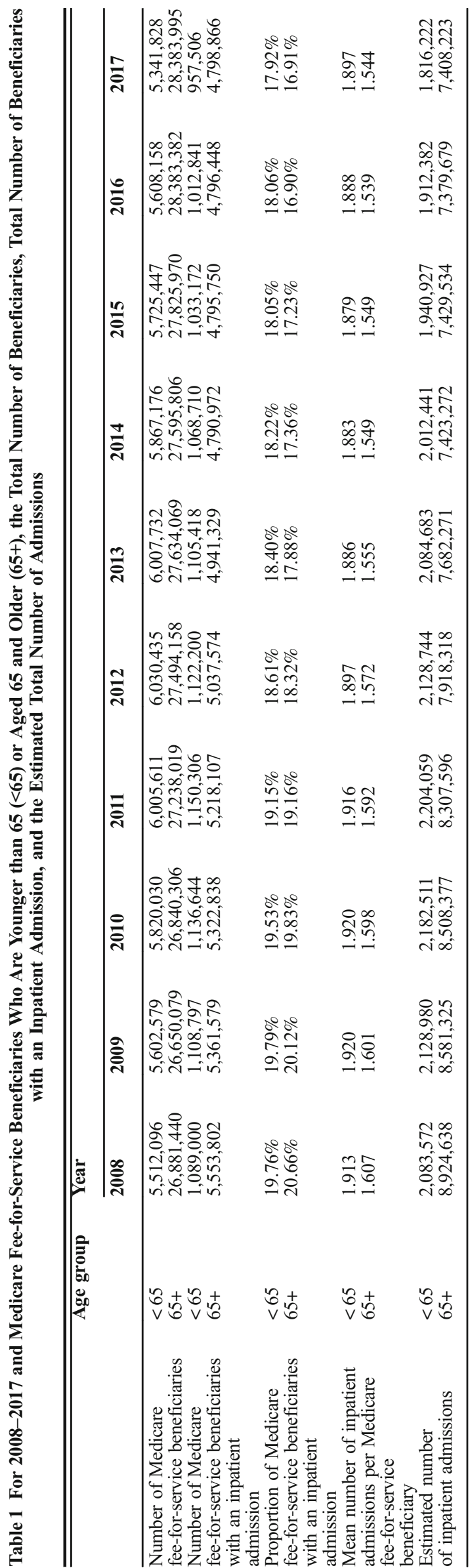

\section{DISCUSSION}

Among Medicare fee-for-service beneficiaries, between 2008 and 2017, overall admission rates fell, peradmission and per-beneficiary ACSC admission rates varied according to the ACSC examined, and ACSC rates per admission were invariably higher than those per beneficiary. ACSC admissions represented a high proportion of all Medicare fee-for-service admissions.

While per-admission and per-beneficiary ACSC admission rates were directionally the same in most cases, we believe that per-admission ACSC rates paint a more accurate and actionable performance picture. A peradmission metric not only reflects community-level performance but also conveys the proportion of potentially wasteful admissions among Medicare fee-for-service beneficiaries, particularly in the context of changing per-capita admission rates. Our analysis suggests that the large reduction in overall Medicare admissions that we saw was not driven by a reduction in wasteful admissions.

Our study was limited by its reliance on national data collected and aggregated by CMS, data restrictions that required us to aggregate PQI data for 65-74 and 75+ populations, and our inability to determine the degree of overlap of readmission and ACSC admission rates. Nonetheless, it demonstrated that opportunities for reducing potentially unwarranted admissions are substantial, and might be more actionable, focused, nuanced, and effective than focusing on readmission rates. ${ }^{5}$ Should those opportunities be addressed-for instance, through re-investment of avoided inpatient care funding into primary care services - further reductions in per-capita inpatient care utilization and costs, and improvements in healthcare value, should occur. 


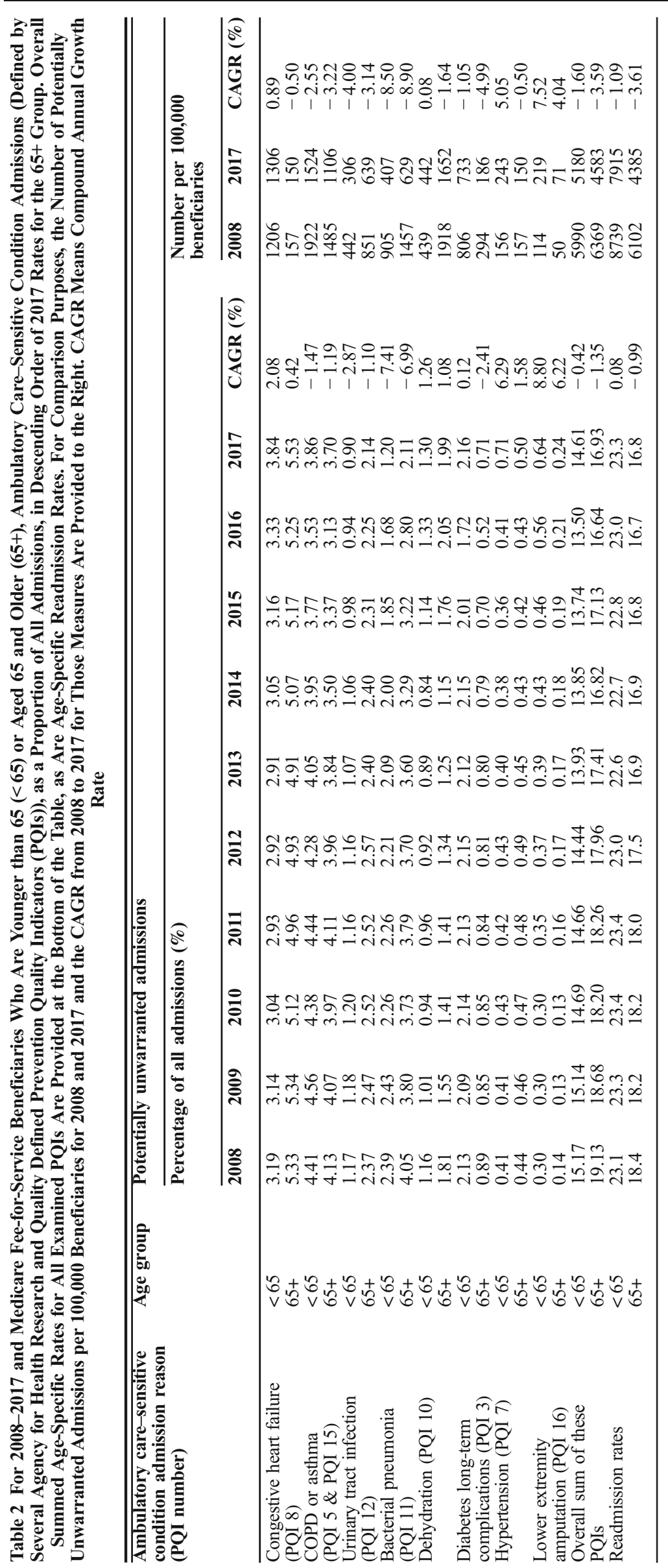


Corresponding Author: William B. Weeks, $M D, P h D, M B A ;$ Microsoft Healthcare NExT, Redmond, WA, USA (e-mail: William. weeks@microsoft.com).

\section{REFERENCES}

1. AHRQ Quality Indicators: Guide to Prevention Quality Indicators. Hospital Admission for Ambulatory Care Sensitive Conditions. http://www.ahrq. gov/downloads/pub/ahrqqi/pqiguide.pdf: Department of Health and Human Services. Agency for Healthcare Research and Quality. Accessed 28 June 2019.

2. Chang $\mathbf{C H}$, Stukel TA, Flood AB, Goodman DC. Primary care physician workforce and Medicare beneficiaries' health outcomes. JAMA 2011;305:2096-105.

3. Weeks WB, Ventelou B, Paraponaris A. Rates of admission for ambulatory care sensitive conditions in France in 2009-2010: trends, geographic variation, costs, and an international comparison. Eur J Health Econ 2016; 17:453-70.

4. Centers for Medicare \& Medicaid Services Public Use files (http://www cms.gov/research-statistics-data-and-systems/statistics-trends-and-reports/medicare-geographic-variation/gv_puf.html) and Original Medicare Enrollment: Part A and/or Part B Enrollees, by Age Group, Calendar years 2008-2017 (http://www.cms.gov/Research-Statistics-Data-and-Systems/Statistics-Trends-and-Reports/CMSProgramStatistics/2013/Enrollment.html): Centers for Medicare \& Medicaid Services (both accessed June 28, 2019).

5. Ody C, Cutler D. The Medical Hospital Readmission Reduction Program: Does It Do Any Good? JAMA Intern Med 2019.

Publisher's Note Springer Nature remains neutral with regard to jurisdictional claims in published maps and institutional affiliations. 